\title{
The Peierls-Hubbard model at weak coupling
}

\author{
Michael Dzierzawa and Carmen Mocanu \\ Institut für Physik, Universität Augsburg, 86135 Augsburg, Germany \\ E-mail: michael.dzierzawa@physik.uni-augsburg.de
}

\begin{abstract}
We investigate the Peierls transition in the one-dimensional PeierlsHubbard model at half filling in the adiabatic approximation for the lattice. Depending on the value of the electron-lattice coupling constant $g$ the equilibrium dimerization can be either enhanced or suppressed by the Hubbard interaction $U$. Applying second order perturbation theory we determine the critical value $g_{c}=0.689348$ below which the Hubbard interaction enhances the dimerization.
\end{abstract}

PACS numbers: 71.10.Fd,71.30.+h

\section{Introduction}

The coupling of a one-dimensional metal to an elastic lattice results in an instability towards a lattice distortion known as Peierls transition [1]. This phenomenon can be observed in various quasi-one-dimensional materials, e.g. conjugated polymers [2] like polyacetylene, charge-transfer salts [3] , or spin-Peierls compounds like $\mathrm{CuGeO}_{3}$.

From a theoretical point of view, a first step towards a quantitative description of the Peierls transition has been made by Su, Schrieffer and Heeger (SSH) 4. However, in their model neither the dynamics of the lattice nor the electron-electron interaction has been taken into account. Subsequently there has been made some effort to include phonons beyond the adiabatic approximation. While in the approach of SSH the Peierls transition occurs for any nonzero value of the electron-phonon (el-ph) coupling, a minimum strength of the el-ph coupling is required for the transition if quantum phonons are included [5].

In this paper we consider the limit of a static lattice and focus on the role of the electron-electron interaction. Correlation effects on the Peierls transition have been studied using a variety of methods including variational wave-functions [6, 7, Hartree Fock plus perturbation theory [8], quantum Monte Carlo [9], numerical diagonalization of small systems [10, 11, 12, 13, bosonization 14, 15, and incremental expansion [16] 17. It is now generally accepted that for small values of the el-ph coupling constant $g<g_{c}$ the dimerization is enhanced when the Hubbard interaction $U$ is switched on while for $g>g_{c}$ it is suppressed. Estimates for the critical el-ph coupling that have been obtained in the literature are $g_{c} \approx 0.75$ in [7, $g_{c} \approx 0.76$ in [12] and $g_{c} \approx 0.69$ in [16, 17. For $g$ not too close to $g_{c}$ the dimerization reaches a maximum at $U$ of the order of the bandwidth and goes to zero for $U \rightarrow \infty$.

Apart from this qualitative agreement there is still a lack of rigorous results, in particular in the limit of weak el-ph coupling $g \ll g_{c}$ which is difficult to address in the aforementioned approaches. In order to remove this uncertainties we study the 
Peierls-Hubbard model in the weak coupling regime using second order perturbation theory with respect to the Hubbard interaction. This allows use to derive rigorous results in the limit $U \rightarrow 0$ for all values of the el-ph coupling.

The paper is organized as follows: In Section two we introduce the PeierlsHubbard model and discuss the validity of the perturbative approach. The main results concerning the Peierls transition are presented in Section three. In Section four we discuss our results and compare them with different approaches.

\section{Model and perturbation theory}

We consider the one-dimensional Peierls-Hubbard model

$$
\begin{aligned}
H= & -t \sum_{i, \sigma}\left(1-\alpha\left(u_{i+1}-u_{i}\right)\right)\left(c_{i \sigma}^{\dagger} c_{i+1, \sigma}+\text { h.c. }\right) \\
& +U \sum_{i} n_{i \uparrow} n_{i \downarrow}+\frac{K}{2} \sum_{i}\left(u_{i}-u_{i+1}\right)^{2}
\end{aligned}
$$

where $c_{i \sigma}^{\dagger}\left(c_{i \sigma}\right)$ creates (annihilates) an electron with spin $\sigma(=\uparrow, \downarrow)$ on a lattice of $N$ sites, $n_{i \sigma}=c_{i \sigma}^{\dagger} c_{i \sigma}$, and $u_{i}$ is the deviation of the $i$ 'th atom from its equilibrium position in units of the lattice constant $a . U$ and $K$ are the on-site Hubbard interaction and the elastic constant of the lattice, respectively, and $t$ is the hopping parameter. In (11) the phonons are treated in the adiabatic limit which is formally obtained by letting the mass of the atoms go to infinity. Since the lattice distortion is assumed small compared to the lattice constant, the modification of the hopping amplitude can be treated in linear order via the parameter $\alpha$. In the following we restrict ourselves to half filling where due to $2 k_{F}=\pi / a$ the Peierls instability leads to an alternating lattice distortion

$$
u_{i}=(-1)^{i} u
$$

It is convenient to express the el-ph coupling and the dimerization amplitude by the dimensionless parameters $g$ and $\delta$, respectively, which are defined by

$$
\begin{aligned}
g & =\alpha \sqrt{\frac{t}{K}} \\
\delta & =2 \alpha u
\end{aligned}
$$

In terms of these parameters Hamiltonian (11) reads

$$
H=-t \sum_{i, \sigma}\left(1+(-1)^{i} \delta\right)\left(c_{i \sigma}^{\dagger} c_{i+1, \sigma}+\text { h.c. }\right)+U \sum_{i} n_{i \uparrow} n_{i \downarrow}+\frac{N t \delta^{2}}{2 g^{2}}
$$

We consider $t, U$ and $g$ as given model parameters while the dimerization $\delta$ is free to adjust itself such that the total energy is minimal. In the following we measure all energies in units of the hopping amplitude $t$ (i.e. we set $t=1$ ). The ground state energy of the Hamiltonian (5) can only be calculated analytically in the limiting cases $\delta=0$ and $\delta=1$, respectively. $\delta=0$ corresponds to the ordinary Hubbard model which has been solved using Bethe ansatz [18 and for $\delta=1$ the system is completely dimerized. For other values of $\delta$ one has to resort to approximations or to numerical methods. Since we are only interested in the weak coupling regime we use second order perturbation theory with respect to the Hubbard interaction. First of all we want to justify the assertion that perturbation theory can indeed be applied 
for the model (5). In the case $\delta=0$ the exact ground state energy known from the Bethe ansatz solution [18 can be expressed as an integral over Bessel functions. As pointed out by Economou and Poulopoulos [19] this integral can be converted into an asymptotic series in $U$. Metzner and Vollhardt 20] showed that the exact second order term of this asymptotic expansion can be calculated using perturbation theory. Furthermore, they conjectured that this is true not only for the second order term but for all coefficients of the expansion. For $\delta>0$ the conditions for applying perturbation theory are more favorable than for $\delta=0$ since the unperturbed ground state energy $E_{0}$ is separated from all excited states by an energy gap $\Delta=4 \delta$ which avoids divergent contributions from small denominators. We expect therefore that the ground state energy of the Peierls-Hubbard model can be expanded in a series with a finite radius of convergence, $U_{0}(\delta)$ for all $\delta>0$ and that the coefficients of this expansion can be obtained from perturbation theory. Krivnov and Ovchinnikov 21] have pointed out that within the parquet approximation for a continuum version of the model (5) the leading n'th order contribution to the ground state energy is $\propto \delta^{2} U^{n} \ln ^{n+1} \delta$. Similar conclusions were obtained by Horovitz and Solyom 22 using a scaling approach and by Kivelson et al [23] by comparison of exact results on the massive Thirring model with the perturbation theory for a model of spinless fermions. Fermions with spin were considered in 24] and again a perturbation expansion in powers of $U \ln \delta$ was obtained. Thus the radius of convergence behaves as $U_{0}(\delta) \propto 1 /|\ln \delta|$ and even for exponentially small dimerization $\delta$ perturbation theory can be applied over some finite range of $U$. Writing $\epsilon(\delta, U)=E(\delta, U) / N$, the ground state energy per site of the electronic part of (5), the expansion of $\epsilon$ in powers of $U$ takes the form

$$
\epsilon(\delta, U)-\frac{U}{4}=\epsilon_{0}(\delta)+\epsilon_{2}(\delta) U^{2}+\epsilon_{4}(\delta) U^{4}+\ldots
$$

where we have used that due to particle-hole symmetry $\epsilon(\delta, U)-U / 4$ is an even function of $U$. In particular, in the case of complete dimerization, $\delta=1$, it is straightforward to calculate the exact ground state energy and expand it in a series which converges for $U<8$ :

$$
\epsilon(1, U)-\frac{U}{4}=-2 \sqrt{1+\frac{U^{2}}{64}}=-2-\frac{U^{2}}{64}+\frac{U^{4}}{16384}+\ldots .
$$

\section{Equilibrium dimerization}

In order to determine the equilibrium dimerization we minimize the ground state energy of the Hamiltonian (5), including the lattice contribution, with respect to $\delta$ and obtain the condition

$$
\epsilon_{0}^{\prime}(\delta)+\epsilon_{2}^{\prime}(\delta) U^{2}+\epsilon_{4}^{\prime}(\delta) U^{4}+\ldots+\frac{\delta}{g^{2}}=0
$$

Eq. (8) defines the function $\delta(U)$ which can as well be expanded in a series containing only even powers of $U$

$$
\delta(U)=\delta_{0}+\delta_{2} U^{2}+\delta_{4} U^{4}+\ldots
$$

Reinserting Eq. (9) into Eq. (8) and comparing order by order in $U$ yields

$$
\begin{aligned}
& \delta_{0}=-g^{2} \epsilon_{0}^{\prime}\left(\delta_{0}\right) \\
& \delta_{2}=-\frac{\epsilon_{2}^{\prime}\left(\delta_{0}\right)}{g^{-2}+\epsilon_{0}^{\prime \prime}\left(\delta_{0}\right)} \\
& \delta_{4}=-\frac{\frac{1}{2} \delta_{2}^{2} \epsilon_{0}^{\prime \prime \prime}\left(\delta_{0}\right)+\delta_{2} \epsilon_{2}^{\prime \prime}\left(\delta_{0}\right)+\epsilon_{4}^{\prime}\left(\delta_{0}\right)}{g^{-2}+\epsilon_{0}^{\prime \prime}\left(\delta_{0}\right)}
\end{aligned}
$$




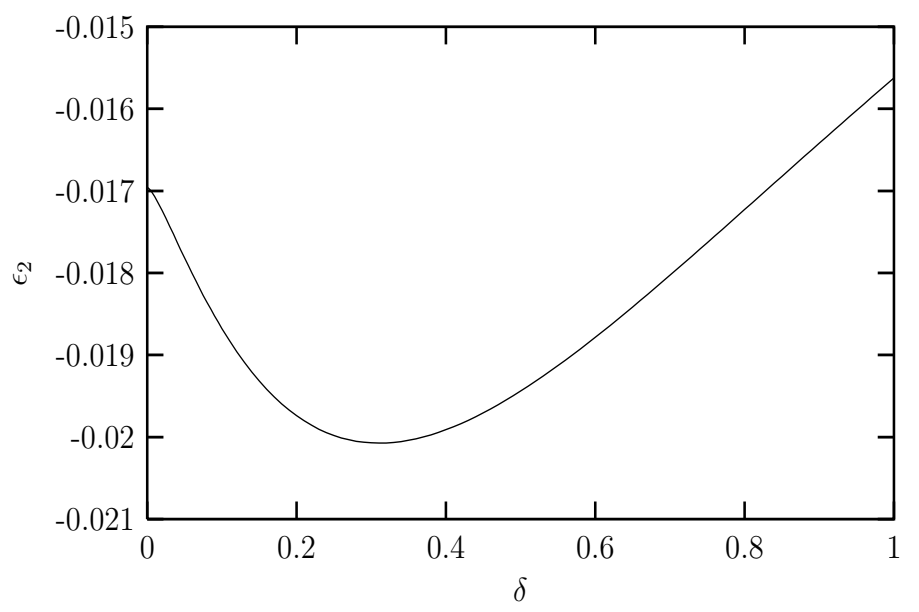

Figure 1. Second order contribution to the ground state energy per site, $\epsilon=\epsilon_{0}+\epsilon_{2} U^{2}+\epsilon_{4} U^{4}+\ldots$, as function of the dimerization parameter $\delta$. The minimum occurs at $\delta_{c}=0.310523$.

The zeroth order dimerization has been calculated in [4]. In the limit $g \ll 1$ it is given by

$$
\delta_{0}=4 \exp \left(-\frac{\pi}{4 g^{2}}-1\right)
$$

Now we turn to the second order contribution $\propto U^{2}$ of the ground state energy. Considering the Hubbard interaction in Eq. (5) as perturbation, the second order coefficient of the series (6) reads

$$
\epsilon_{2}(\delta)=-\frac{1}{N} \sum_{n \neq 0} \frac{|\langle n|D| 0\rangle|^{2}}{E_{n}-E_{0}}
$$

where $E_{n}$ is the energy of the eigenstate $|n\rangle$ of the noninteracting Hamiltonian $H_{0}$, and $D=\sum_{i} n_{i \uparrow} n_{i \downarrow}$. Representing $|n\rangle$ in the one-particle basis of $H_{0}$, Eq. (14) can be converted into

$$
\epsilon_{2}(\delta)=-\frac{1}{32} \int_{-\pi}^{\pi} \frac{\mathrm{d} k}{2 \pi} \int_{-\pi}^{\pi} \frac{\mathrm{d} k^{\prime}}{2 \pi} \int_{-\pi}^{\pi} \frac{\mathrm{d} q}{2 \pi} \frac{\left|1+\mathrm{e}^{i\left(\varphi_{k+q}-\varphi_{k}+\varphi_{k^{\prime}-q}-\varphi_{k^{\prime}}\right)}\right|^{2}}{\varepsilon_{k+q}+\varepsilon_{k}+\varepsilon_{k^{\prime}-q}+\varepsilon_{k^{\prime}}}
$$

where

$$
\begin{aligned}
\varepsilon_{k} & =2 \sqrt{\cos ^{2} k+\delta^{2} \sin ^{2} k} \\
\mathrm{e}^{i \varphi_{k}} & =\frac{1+\mathrm{e}^{-2 i k}+\delta\left(1-\mathrm{e}^{-2 i k}\right)}{\left|1+\mathrm{e}^{-2 i k}+\delta\left(1-\mathrm{e}^{-2 i k}\right)\right|} .
\end{aligned}
$$

Eq. (15) is equivalent with Eq. (C5) in Ref. 8 in the limit of zero staggered magnetization. The integral (15) can be calculated numerically with very high precision. The result shown in Fig. 1 agrees with the analytical values $\epsilon_{2}(0)=$ $-\left(7 / 16 \pi^{3}\right) \zeta(3)=-0.016961$ and $\epsilon_{2}(1)=-1 / 64=-0.015625$. More importantly, $\epsilon_{2}(\delta)$ has a minimum at $\delta_{c}=0.310523$ which means that according to Eq. (11) the sign of the coefficient $\delta_{2}$ changes from plus to minus. According to Eq. (10) the critical dimerization $\delta_{c}$ corresponds to a critical el-ph coupling constant

$$
g_{c}=\sqrt{\frac{\delta_{c}}{\left|\epsilon_{0}^{\prime}\left(\delta_{c}\right)\right|}}=0.689348
$$




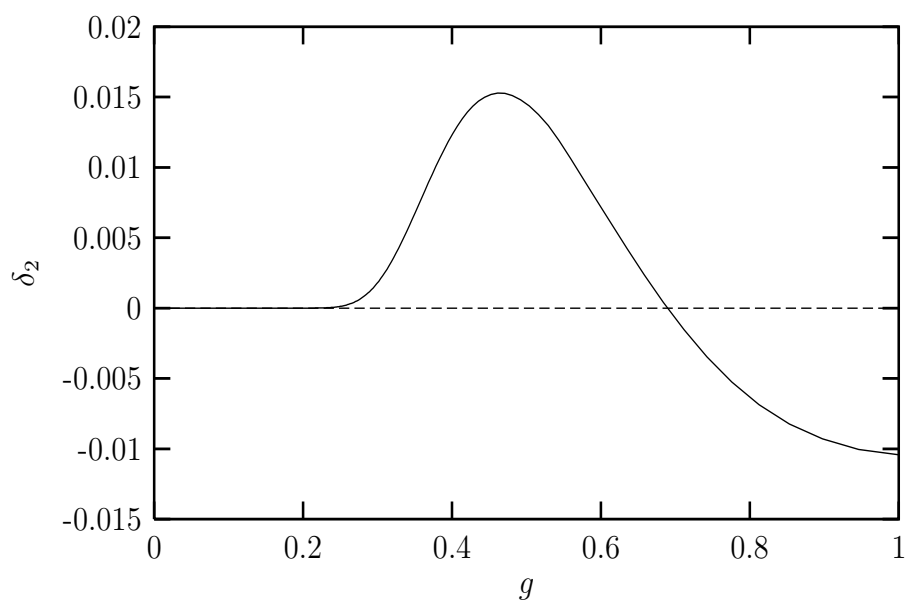

Figure 2. Second order coefficient $\delta_{2}$ of the equilibrium dimerization $\delta(U)=$ $\delta_{0}+\delta_{2} U^{2}+\delta_{4} U^{4}+\ldots$ as function of the el-ph coupling constant $g$. Note that $\delta_{2}$ changes sign at $g_{c}=0.689348$ which is related to $\delta_{c}=0.310523$ via Eq. [18].

Therefore for $g<g_{c}$ the dimerization is enhanced by the Hubbard interaction while for $g>g_{c}$ it is suppressed. The second order coefficient $\delta_{2}$ of Eq. (9) which is displayed in Fig. [2] has a maximum at $g \approx 0.464$. In the limit of small el-ph coupling, $g \ll 1$, Eq. (111) simplifies to

$$
\delta_{2}=-\frac{\pi}{4} \epsilon_{2}^{\prime}\left(\delta_{0}\right)
$$

From a careful analysis of the numerical data for small values of $\delta$ we conjecture that

$$
\epsilon_{2}(\delta)-\epsilon_{2}(0) \propto \delta^{2}\left(\ln \frac{c}{\delta}\right)^{3}
$$

with $c \approx 1.5$, in agreement with the result of the parquet summation 21]. Inserting this expression into Eq. (19) and using the asymptotic formula for $\delta_{0}(g)$ given in Eq. (13) we obtain the result

$$
\frac{\delta_{2}}{\delta_{0}} \propto g^{-6}
$$

i.e. the relative weight of the second order term in the expansion of the dimerization $\delta(U)=\delta_{0}+\delta_{2} U^{2}+\delta_{4} U^{4}+\ldots$ increases strongly for $g \rightarrow 0$, although both $\delta_{0}$ and $\delta_{2}$ are exponentially small in this limit.

Unfortunately it becomes rather complicated to calculate the fourth order term $\epsilon_{4}(\delta)$ using perturbation theory. We have therefore chosen a different approach based on the exact numerical diagonalization of small systems (up to $L=14$ lattice sites) using the Lanczos algorithm which allows us to calculate the ground state energy with a relative precision of better than $10^{-14}$. In order to avoid even-odd oscillations we have implemented antiperiodic boundary conditions for $L=4 n$ and periodic boundary conditions for $L=4 n+2$. This guarantees that the ground state is non-degenerate. Since we know the exact second order term $\epsilon_{2}(\delta, L)$ (for given system size $L$ ) it is possible to extract $\epsilon_{4}(\delta, L)$ with very high precision. However, one is left with the problem of extrapolating these values to $L=\infty$. We have tried various extrapolation procedures. For $\delta \geq 0.2$ the ansatz $\epsilon_{4}(\delta, L)=\epsilon_{4}(\delta, \infty)+a \exp (-b L+c / L)$, which 


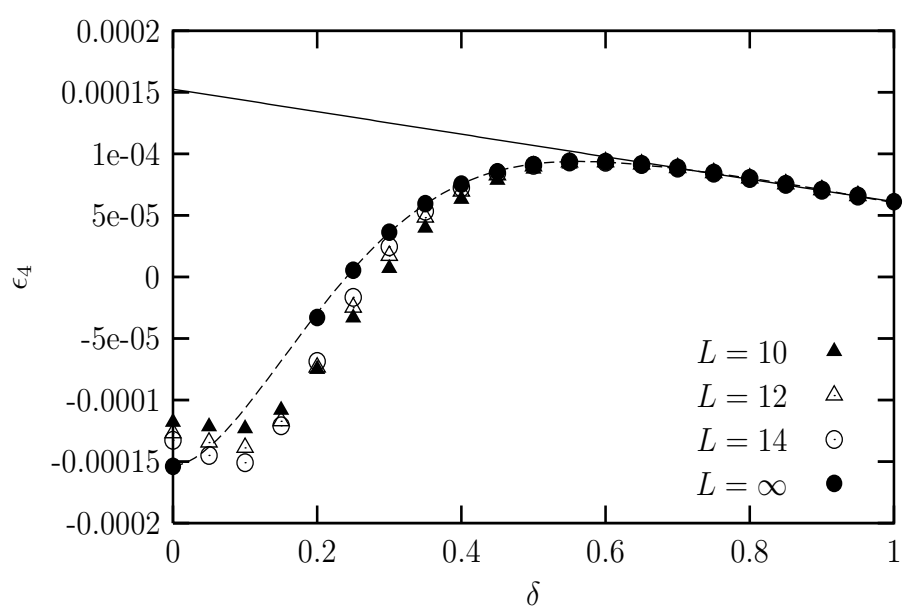

Figure 3. Fourth order contribution of the ground state energy per site, $\epsilon(L)=\epsilon_{0}(L)+\epsilon_{2}(L) U^{2}+\epsilon_{4}(L) U^{4}+\ldots$, as function of the dimerization parameter $\delta$ for $L=10,12,14$ and $\infty$. The dashed curve is meant as a guide to the eye, only. The solid line is the asymptotic result $\epsilon_{4}(\delta)=2^{-14}(1+3(1-\delta) / 2)$ valid in the limit $\delta \rightarrow 1$.

is motivated by the existence of a gap in the excitation spectrum, yields very stable results for $\epsilon_{4}(\delta, \infty)$. In particular, the asymptotic behavior close to the fully dimerized limit, $\epsilon_{4}(\delta)=2^{-14}(1+3(1-\delta) / 2+\ldots)$ for $\delta \rightarrow 1$ is correctly recovered (see Fig. 3). However, for $\delta<0.2$ we could not perform a reliable finite-size scaling of our data. The reason is that the correlation length, which is inversely proportional to the gap, is getting much larger than the size of the systems that can be diagonalized numerically. On the other hand, for $\delta=0$ where there is no gap, an unbiased polynomial fit yields $\epsilon_{4}(0, \infty)=-1.547 \times 10^{-4}$ which is quite close to the exact value [19] $\epsilon_{4}(0)=-\left(93 / 2048 \pi^{5}\right) \zeta(5)=-1.538 \times 10^{-4}$. In Fig. 3] we plot $\epsilon_{4}(0, L)$ for $L=10,12,14$ together with the extrapolated value $\epsilon_{4}(0, \infty)$ (whenever available) as a function of $\delta$. Comparing with the corresponding results for $\epsilon_{2}$ (see Fig. 11) we see that $\epsilon_{4}$ is typically two orders of magnitude smaller than $\epsilon_{2}$, which indicates that the weak coupling approach should be quite accurate in the region $U \lesssim 1$.

For $g<g_{c}$ the dimerization $\delta(U)$ reaches a maximum at $U=U_{\max }$ which is given by

$$
U_{\max }=\sqrt{-\frac{\delta_{2}}{2 \delta_{4}}}
$$

when we neglect the terms of order $\sim U^{6}$ and higher in Eq. (9). Expanding $U_{\max }^{2}$ in powers of $g_{c}-g$ we obtain for $g$ close to $g_{c}$

$$
U_{\max }(g) \simeq \kappa\left(g_{c}-g\right)^{1 / 2}
$$

with

$$
\kappa=\frac{\delta_{c} \epsilon_{2}^{\prime \prime}\left(\delta_{c}\right)}{g_{c} \epsilon_{4}^{\prime}\left(\delta_{c}\right)\left(1+g_{c}^{2} \epsilon_{0}^{\prime \prime}\left(\delta_{c}\right)\right)} \approx 8.7
$$

to be compared with the value $\kappa=8.25$ obtained in Ref. [16] using the incremental expansion. Note that the error in the determination of $\kappa$ is only due to the limited 


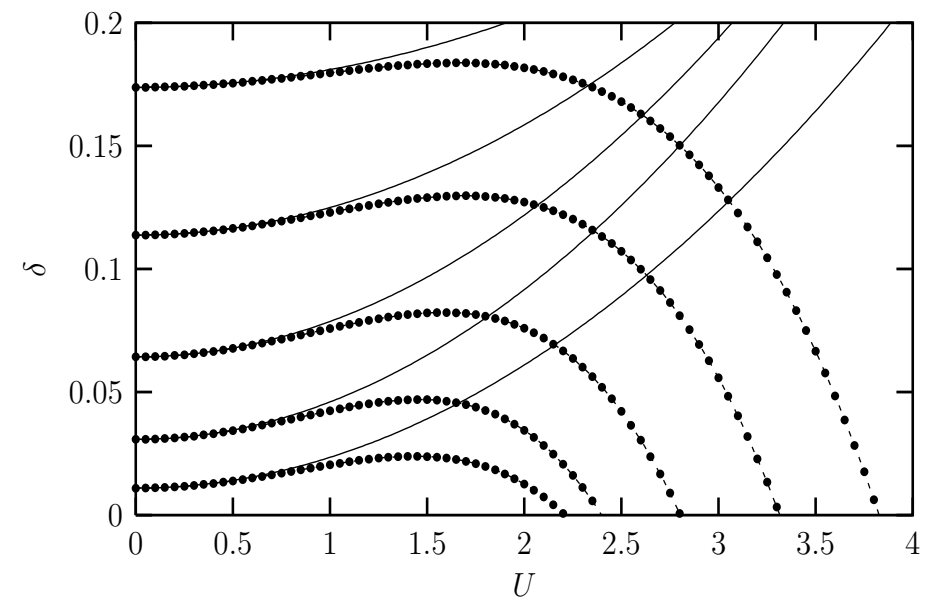

Figure 4. Dimerization $\delta$ as function of $U$ for $g=0.4,0.45,0.5,0.55,0.6$ (from bottom to top). The full lines (circles) represent the second (fourth) order approximation of $\delta(u)=\delta_{0}+\delta_{2} U^{2}+\delta_{4} U^{4}+\ldots$ The values of $U$ where $\delta$ becomes negative indicate the breakdown of the weak coupling approximation.

precision of $\epsilon_{4}$. Nevertheless, the fair agreement with the result of [16] indicates that the finite-size scaling of our Lanczos data yields reasonable results for $\epsilon_{4}$.

In Fig. 4 the equilibrium dimerization $\delta$ of the Peierls-Hubbard model is displayed as function of $U$ for several values of the el-ph coupling $g$. The circles represent the dimerization including the fourth order term in $U$ while the solid curves correspond to the second order approximation. For small values of $g$ the maximum of $\delta$ is more pronounced and occurs around $U \approx 2$. This is somewhat smaller than the value $U \approx 3$ obtained using the incremental expansion [16. Furthermore the maximum of $\delta$ is expected to shift to larger values of $U$ with decreasing $g$ but of course the precise position of the maximum depends on the terms of order $\propto U^{6}$ and higher which have been omitted in our weak coupling approach.

\section{Discussion}

We have investigated the Peierls Hubbard model in the weak-coupling regime using second order perturbation theory and exact diagonalization for the fourth order contribution. The application of perturbation theory is justified by the existence of a gap in the excitation spectrum which removes the problem of small denominators. On the other hand, it is also due to this gap that finite-size scaling of the Lanczos data becomes problematic for small dimerization $\delta<0.2$ where the correlation length exceeds the size of the systems that can be diagonalized numerically. The central result of this paper is the determination of the exact value of the el-ph coupling $g_{c}=0.689348$ which separates the region where the Hubbard interaction enhances the dimerization $\left(g<g_{c}\right)$ from the region where the dimerization is reduced $\left(g>g_{c}\right)$. The corresponding dimerization parameter is found to be $\delta_{c}=0.310523$. There have been many attempts to determine these parameters using different methods. Using the Gutzwiller variational wave-function Baeriswyl and Maki [7] estimated $g_{c} \approx 0.75$. Exact diagonalization of small systems has been used to obtain $\delta_{c} \approx 0.4$ in [11] and $g_{c} \approx 0.76$ in [12, respectively. Finally, the most precise values up to now have been 
derived using the incremental expansion technique, which is based on the numerical diagonalization of finite chains. In [16 17 $g_{c}=0.69$ is obtained, which is very close to the exact value.

As mentioned before, all approaches that rely on finite-size extrapolation of small systems suffer from severe problems in the limit of small dimerization, $\delta<0.1$, which corresponds to values of the el-ph coupling $g<0.5$. In contrast, the perturbation theory approach can be applied for all values of $g$. In particular, it is possible to determine the asymptotic behavior of $\epsilon_{2}(\delta)$ in the limit $\delta \rightarrow 0$ including logarithmic corrections. From there it can be inferred that the relative importance of the second order term (compared to the leading term) in the expansion of the dimerization $\delta(U)=\delta_{0}+\delta_{2} U^{2}+\delta_{4} U^{4}+\ldots$ goes as $\sim g^{-6}$ for $g \rightarrow 0$, although both $\delta_{0}$ and $\delta_{2}$ are exponentially small in this limit. On the other hand, considering absolute values, the correlation enhancement of the dimerization is less dramatic and essentially limited to the region $0.3<g<g_{c}$ with the maximum occurring at $g=0.464$. The parameters most frequently used for polyacetylene lie in the range between $g=0.39$ [7] and $g=0.57$ [4] which emphasizes the importance of correlation effects in real Peierls systems.

\section{Acknowledgements}

We thank C Schuster, P Schwab, U Eckern, and H Zheng for helpful discussions. This work was supported by the Deutsche Forschungsgemeinschaft (SFB 484).

\section{References}

[1] Peierls R E 1955 Quantum Theory of Solids (Oxford: Oxford University Press)

[2] Kiess H G 1992 Conjugated Conducting Polymers (Berlin: Springer)

[3] Ishiguro T and Yamaji K 1990 Organic Superconductors (Berlin: Springer)

[4] Su W P, Schrieffer J R and Heeger A J 1979 Phys. Rev. Lett. 421698 Su W P, Schrieffer J R and Heeger A J 1980 Phys. Rev. B 222099

[5] Sengupta P, Sandvik A W and Campbell D K 2003 Phys. Rev. B 67245103

[6] Horsch P 1981 Phys. Rev. B 247351

[7] Baeriswyl D and Maki K 1985 Phys. Rev. B 316633

[8] Kivelson S and Heim D E 1982 Phys. Rev. B 264278

[9] Hirsch J E 1983 Phys. Rev. Lett. 51296

[10] Mazumdar S and Dixit S N 1983 Phys. Rev. Lett. 51292

[11] Hayden G W and Soos Z G 1988 Phys. Rev. B 386075

[12] Waas V, Büttner H and Voit J 1990 Phys. Rev. B 419366

[13] Ogata M 1993 Prog. Theor. Phys. Supp. 113215

[14] Sugiura M and Suzumura Y 2002 J. Phys. Soc. Jpn. 71697

[15] Mocanu C, Dzierzawa M, Schwab P and Eckern U 2004 J. Phys. : Condens. Matter 166445 Mocanu C, Dzierzawa M, Schwab P and Eckern U 2005 Phys. Status Solidi b 242245

[16] Málek J, Kladko K and Flach S 1998 JETP Lett. 671052

[17] Málek J, Drechsler S-L, Flach S, Jeckelmann E and Kladko K 2003 J. Phys. Soc. Jpn. 722277

[18] Lieb E H and Wu F Y 1968 Phys. Rev. Lett. 201445

[19] Economou E N and Poulopoulos P N 1979 Phys. Rev. B 204756

[20] Metzner W and Vollhardt D 1989 Phys. Rev. B 394462

[21] Krivnov V Ya and Ovchinnikov A A 1984 JETP Lett. 39 159; 1986 Zh. Eksp. Teor. Fiz. 90709

[22] Horovitz B and Sólyom J 1985 Phys. Rev. B 322681

[23] Kivelson S, Thacker $\mathrm{H}$ and Wu W-K 1985 Phys. Rev. B 313785

[24] Wu W-K and Kivelson S 1986, Phys. Rev. B 338546 